\title{
5. Sexually Transmitted Infections
}

\section{Kaposi sarcoma lesions respond differently to therapy in HIV- infected and noninfected patients}

Guedes F, de Andrade HF Jr, Fernandes ER, et al. The effects of human herpesvirus 8 infection and interferon- $\gamma$ response in cutaneous lesions of Kaposi sarcoma differ among human immunodeficiency virus-infected and uninfected individuals.

Br J Dermatol 2008;159(4):839-846

doi:10.1111/j.1365-2133.2008.08755.x

Objective: To evaluate the human herpesvirus 8 (HHV-8) expression and immune response in cutaneous lesions of classic KS (CKS) and AIDS-associated KS (AIDS-KS).

Background and methods: Kaposi sarcoma (KS) is associated with HHV-8. The cutaneous immune response in this tumor is not well established and a better understanding is necessary. A quantitative immunohistochemical study was performed of cells expressing HHV-8 latency-associated nuclear antigen (LANA), CD4, CD8, and interferon (IFN)- $\gamma$ in skin lesions from patients with CKS and AIDS-KS (with or without highly active antiretroviral therapy [HAART]).

Result: CKS showed higher LANA expression than AIDS-KS, regardless of HAART. Higher LANA expression was also found in nodules than patch/plaque lesions. The tissue CD4 + cell proportion was lower in AIDS-KS patients without HAART than in patients with CKS. In CKS lesions, CD4 + and CD8 + cells expressed IFN- $\gamma$, as shown by double immunostaining. AIDS-KS presented low numbers of IFN- $\gamma$-expressing cells. CD8 + cell numbers were similar in all groups, which appeared unrelated to the clinical or epidemiological type of KS.

Conclusion: The quantitative data on the pattern of KS lesions in selected groups of patients, as shown by in situ immune response, demonstrated a CD4 + T-cell involvement associated with IFN- $\gamma$, an environment of immune response-modified human immunodeficiency virus (HIV) infection. The promotion of KS in patients without HIV appeared to be related to higher HHV-8 load or virulence than in those with AIDS. This higher resistance may be explained by a sustained immune response against this herpesvirus, that is only partially restored but effective after HAART.

Reprint requests to Dr. Guedes, Department of Pathology, Faculty of Medical Sciences, University of São Paulo, São Paulo 01246-903, Brazil.

\section{ESSENCE}

Penile intraepithelial neoplasia is frequent in HIV-positive men with anal dysplasia

Kreuter A, Brockmeyer NH, Weissenborn SJ, Gambichler T, Stücker M, et al.

J Invest Dermatol 2008;128:2316-2324

doi:10.1038/jid.2008.72

Anogenital human papillomavirus (HPV)-infection is common in HIV-infected men who have sex with men (HIV + MSM). These patients have a strongly increased risk of HPV-induced anal cancer and its precursor lesion, anal intraepithelial neoplasia (AIN), and a moderately increased risk for penile cancer. Only limited data exist on penile intraepithelial neoplasia (PIN) in HIV + MSM. This study determined the prevalence and evaluated the virologic characteristics of PIN and AIN in 263 HIV + MSM. In case of histologically confirmed PIN (and AIN), HPV-typing, HPV-DNA load determination, and immunohistochemical staining for p $16^{\mathrm{INK} 4 \mathrm{a}}$ were performed. PIN was detected in $11(4.2 \%)$ and AIN in $156(59.3 \%)$ patients. Ten PIN patients also had AIN within the observation period. Four clinical types of PINs could be distinguished. High-risk-HPV-DNA was found in 10 PIN lesions, with HPV16 being the most frequent type. Infections with multiple HPV-types were common. All high-grade lesions had high-riskHPV-DNA-loads $\geq 1 \mathrm{HPV}$-copy/ $\beta$-globin-gene-copy. In conclusion, all HIV + MSM should be screened for PIN in addition to AIN screening. 